\title{
Spike Detection in Epileptic Patients EEG Data using Template Matching Technique
}

\author{
$\mathrm{K}$ Vijayalakshmi \\ Department of Medical Electronics, \\ B M S College of Engineering, \\ Bangalore, Karnataka -560019 \\ India
}

\author{
Appaji M Abhishek \\ Department of Medical Electronics, \\ BMS College of Engineering, \\ Bangalore, Karnataka - 560019 \\ India
}

\begin{abstract}
Electroencephalogram (EEG) is the recording of electrical activity along the scalp produced by the firing of neurons within the brain.

It is one of the most important physiological parameter, which is being extensively used for knowing the state of neurological disorder patients. In this work, a program for EEG spike detection has been developed. This program is based on Template Matching Algorithm. The EEG data is taken and a template of EEG spike is taken by manual detection from the EEG data. This template is used by the EEG spike detection program to find spike/spikes in EEG signals using the Template Matching Algorithm. Furthermore, a threshold value is assigned to make a decision because of the variation of amplitude in EEG pattern from person to person. It is marked as spike if the algorithm produces a value more than threshold. The output gives the spike/spikes \& also the position of the sample having the spikes [1].
\end{abstract}

\section{General Terms}

Biomedical Signal Processing.

\section{Keywords}

EEG, Events, Epileptic Patients, Template Matching, Spikes.

\section{INTRODUCTION}

Since the first recording from humans performed in 1929, the EEG has become one of the most important diagnostics tools in clinical neurophysiology, but up to now, EEG analysis still relies mostly on its visual inspection. Due to the fact that visual inspection is very subjective and hardly allows any statistical analysis or standardization, several methods were proposed in order to quantify the information of the EEG.

The objective of this study is to develop a program that will help neurologists to decide the spike waves by analysing the mental problems in the EEG records. The mental disorder taken into consideration in the study is epilepsy. Spike waves of EEG signals have been analysed with Template Matching Algorithm. Template Matching Algorithm may be defined as the pattern to be recognized is compared with a learned template allowing changes in scale. It has been studied to develop a program for the EEG spike detection using the Template
Matching Method. Several methods have been developed previously, but up to now any of them has not certain solution for the spike detection. Spike waves have $20-70$ msec duration. The spikes may occur at any channel from different positions taken from the scalp having different shapes. These spikes may or may not have same duration and shape. However muscle activities can be look like a spike. These activities called "artifacts". Because of these difficulties, each solution works with a performance. The program is implemented on the 10 epileptic data \& arranged in a table for comparative study [2].

\section{EEG SIGNALS}

EEG is the abbreviation for electroencephalography. The aim of electroencephalography is to record and measure samples of electromagnetic fields during certain states and sequences of behavior, in order to explain some of the mechanisms by which behavior is generated. The EEG signals represent electrical changes of the brain during its function. Modern advances in EEG have included what is referred to as digital EEG or dEEG. Here, brain signals are similarly collected from the scalp and amplified but are fed into a computer (i.e., digitized) and then interpreted by viewing them not on paper but on the computer screen. Important advantages include storage of efficient digital media rather than on bulky paper. Another advantage is the ability to view the same EEG signals from different perspectives - paper affords only one view of a time period. A draw-back is that the computer screen may not afford the same clarity of image that is available on paper.

For Measurement Small, non-invasive electrodes (usually 16 to 32 in number) are placed upon a patient's scalp, after careful measurement by a trained technologist, with paste or glue like substance to hold them in place. Low voltage signals (5-500 micro volts) are amplified by the EEG machine and results are typically written by ink-fed pens on a moving paper strip chart.

Typically the EEG is screened for features that stand out (transient responses) like the spike or spike and wave associated with epilepsy. Next, the frequency or spectral content of the remaining EEG background is visually evaluated. There are four broad spectral band of clinical interest; delta $(0-4 \mathrm{~Hz})$, 
theta $(4-8 \mathrm{~Hz})$, alpha $(8-12 \mathrm{~Hz})$,

beta (above $12 \mathrm{~Hz}$ )

Pathology typically increases slow activity (delta, theta) and diminishes fast activity (alpha, beta). Thus, overlying a localized brain tumor one would expect increased slowing and decreased fast activity. However, EEG interpretation requires considerable skill and often years of clinical experience.

\section{TEMPLATE MATCHING ALGORITHM}

Template matching is a technique used to isolate certain features in an image. These features can be single pixels, lines, edges or complete objects. It is easier to look upon an image, convolved with a template window, as a correlation between an image and that window. The result will be an image with high values where there is a strong correlation (that is, where the template matches part of the image) and low values elsewhere. Template Matching is the term given to the process of detecting an event buried in a signal by comparing it to a predefined "template". This template is got by manual detection of peak (Spike) in the EEG data in this research [3].

Even though there are many other methods similar to Template matching technique like 'Stereo matching', 'Image registration' and 'Scale-invariant feature transform' we have selected template matching technique because complexity is less and marking of the spikes can be done easily.

\section{IMPLEMENTATION}

The method first estimates the template by detecting the most prominent spikes. These template/templates are the shape that will be used by the rest of the algorithm to compare the signal against (using one of the two previous mentioned measures). A threshold is then applied to the output of the measured template function, much like a normal threshold detector, the result of which is used to identify detection events. The key difference between a threshold detector and a template based detector is over which function the threshold is applied. With normal threshold detectors, if the amplitude of the incoming data exceeds a threshold, event detection is declared. With a template based detector if the output of the template error measure crosses a threshold, event detection is declared [4].

In order to implement spike detection program, we need to select a suitable segment of EEG Data. In our experiment, the implementing data were selected through a short sampling window and all EEG signals were visually examined by qualified EEG technologist.

This width is that effective way to cover all spike forms. A spike has a pointed peak and duration of 20 to 70 msec.

Template matching was performed using normalized correlation coefficient defined as

$$
Y_{x y}(k)=\frac{\sum_{n=0}^{N}-1[x(n)-3][y(n-k)-y]}{\sqrt{\left.\sum_{n=0}^{N-1}[x(n)-3]^{2} \sum_{n=0}^{N-1} y(n-k)-y\right]^{2}}}
$$

where $\mathrm{x}$ is the template, $\mathrm{y}$ is the EEG signal, $\bar{x}$ and $\bar{y}$ are the averages of the corresponding signals over the $\mathrm{N}$ samples considered, and $\mathrm{k}$ is the time index of the signal $\mathrm{y}$ at which the template is placed.

\section{RESULTS}

The programs was developed and executed.

The following results were obtained.

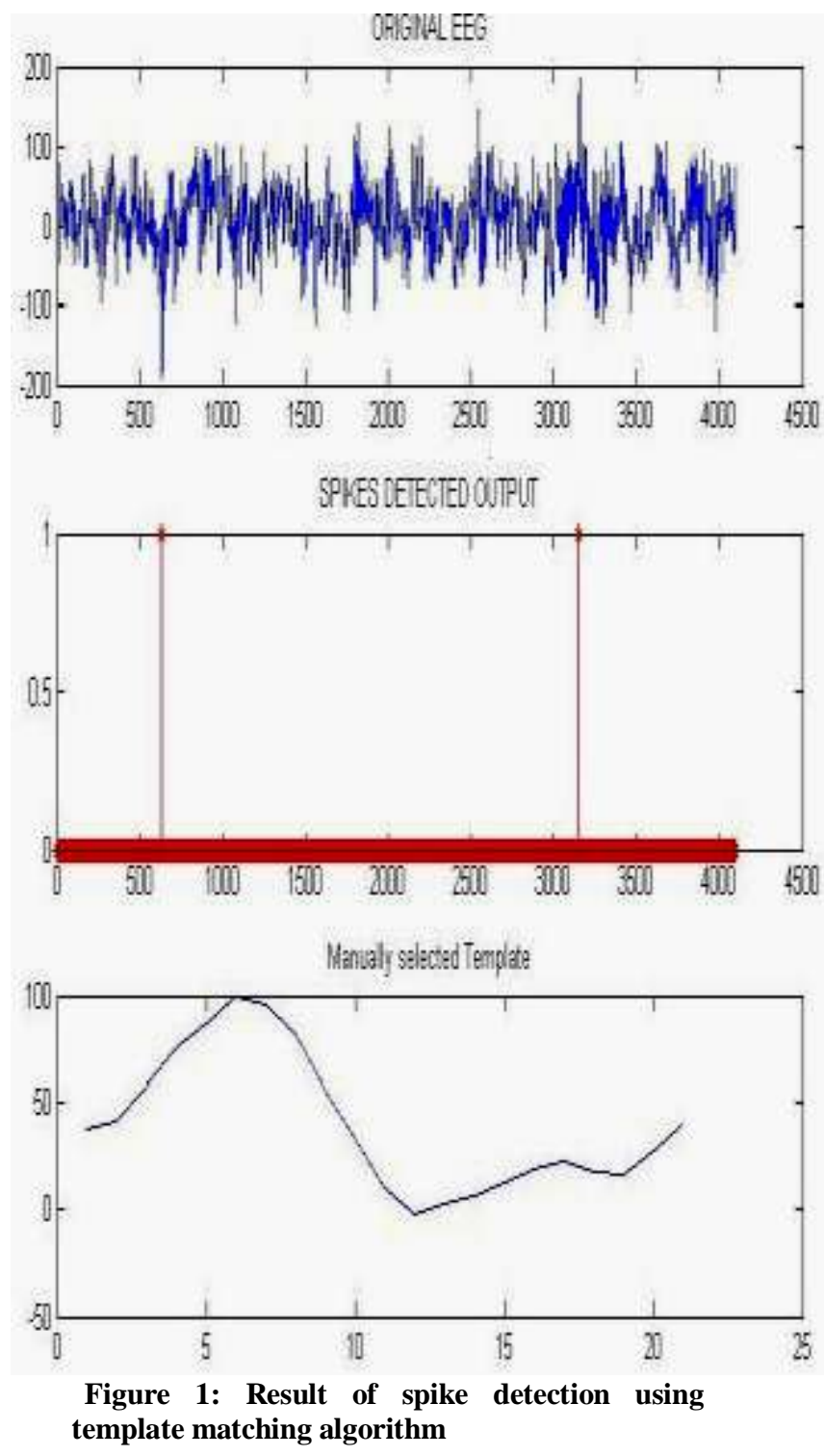

For the threshold of 0.5 and the template range of 3150:3200, the spikes are seen at the locations

$\begin{array}{lllll}629 & 3146 & 3147 & 3148 & 3149\end{array}$


Table1: Result Analysis for 10 data set

\begin{tabular}{|c|c|c|c|c|}
\hline $\begin{array}{c}\text { EEG } \\
\text { data }\end{array}$ & $\begin{array}{c}\text { Manually } \\
\text { selected } \\
\text { Template } \\
\text { Range }\end{array}$ & $\begin{array}{c}\text { No. of } \\
\text { spikes set } \\
\text { detected } \\
\text { (Template } \\
\text { Algorithm) }\end{array}$ & $\begin{array}{c}\text { No. of } \\
\text { spikes } \\
\text { set } \\
\text { expected }\end{array}$ & Difference \\
\hline Data1 & $3950: 4000$ & 1 & 1 & 0 \\
\hline Data2 & $2950: 3000$ & 24 & 23 & 1 \\
\hline Data3 & $1650: 1700$ & 1 & 1 & 0 \\
\hline Data4 & $2650: 2700$ & 7 & 8 & -1 \\
\hline Data5 & $100: 120$ & 8 & 9 & -1 \\
\hline Data6 & $3400: 3500$ & 1 & 2 & -1 \\
\hline Data7 & $1000: 1050$ & 4 & 4 & 0 \\
\hline Data8 & $3360: 3380$ & 15 & 15 & 0 \\
\hline Data9 & $1600: 1630$ & 15 & 15 & 0 \\
\hline Data10 & $3620: 3650$ & 7 & 7 & 0 \\
\hline
\end{tabular}
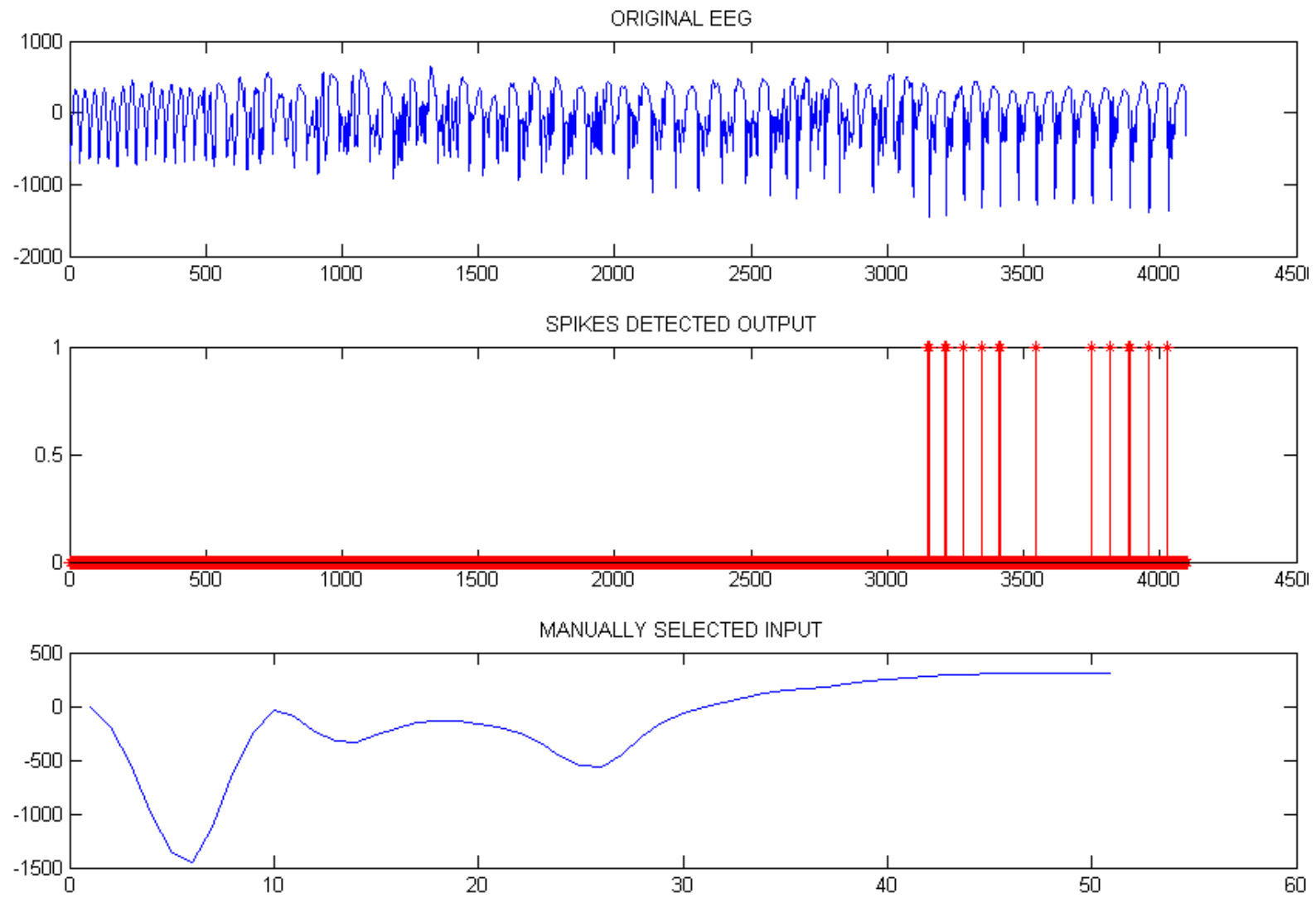

Figure 2 : Result of spike detection using template matching algorithm for Data 2

\begin{tabular}{|c|c|c|c|c|c|c|c|c|c|c|c|}
\hline 214 & 370 & 507 & 783 & 1156 & 1439 & 1791 & 1792 & 2323 & & & \\
\hline 2781 & 3370 & 3712 & 3716 & 3892 & 3893 & & & & & & \\
\hline
\end{tabular}


